\title{
Alterstice
}

Revue internationale de la recherche interculturelle

International Journal of Intercultural Research

Revista International de la Investigacion Intercultural

\section{Accueillir des jeunes réfugiés en région : la formation générale aux adultes comme alternative scolaire?}

\section{Marilyn Steinbach, Michèle Vatz-Laaroussi et Maryse Potvin}

Volume 5, numéro 2, 2015

URI : https://id.erudit.org/iderudit/1036695ar

DOI : https://doi.org/10.7202/1036695ar

Aller au sommaire du numéro

Éditeur(s)

Alterstice

ISSN

1923-919X (numérique)

Découvrir la revue

Citer cet article

Steinbach, M., Vatz-Laaroussi, M. \& Potvin, M. (2015). Accueillir des jeunes réfugiés en région : la formation générale aux adultes comme alternative scolaire? Alterstice, 5(2), 99-108. https://doi.org/10.7202/1036695ar
Résumé de l'article

À partir d'une étude plus large menée auprès de six commissions scolaires et 21 centres d'éducation des adultes (CEA) du Québec, nous avons choisi de présenter dans cet article la situation des jeunes réfugiés qui fréquentent le CEA de la commission scolaire de Sherbrooke, une région hors de la métropole montréalaise. À partir de leur trajectoire scolaire, nous explorons leur accueil dans le CEA, les facteurs qui influencent leur parcours scolaire, les pratiques institutionnelles mises en oeuvre par le monde scolaire à leur égard, les processus suivis à l'intérieur du centre et la manière dont les acteurs institutionnels perçoivent ces jeunes immigrants. Les jeunes rencontrés ont un profil d'apprenants en difficulté ou à risque en raison de leur trajectoire migratoire et scolaire, de leur situation familiale, de leur grand retard scolaire et des traumatismes vécus, mais ils sont résilients au regard des nombreux obstacles situationnels, informationnels, dispositionnels et institutionnels qu'ils rencontrent. Contrairement à la situation des jeunes immigrants montréalais, les jeunes réfugiés en région semblent ainsi utiliser pleinement le contexte et les ressources des CEA comme tuteurs de résilience.
Droits d'auteur (c) Marilyn Steinbach, Michèle Vatz Laaroussi et Maryse Potvin, 2015
Ce document est protégé par la loi sur le droit d'auteur. L'utilisation des services d’Érudit (y compris la reproduction) est assujettie à sa politique d'utilisation que vous pouvez consulter en ligne.

https://apropos.erudit.org/fr/usagers/politique-dutilisation/ 
ARTICLE HORS THÈME

\title{
Accueillir des jeunes réfugiés en région : la formation générale aux adultes comme alternative scolaire?
}

\author{
Marilyn Steinbach ${ }^{1}$, Michèle Vatz-Laaroussi ${ }^{1}$ et Maryse Potvin ${ }^{2}$
}

\section{Résumé}

À partir d'une étude plus large menée auprès de six commissions scolaires et 21 centres d'éducation des adultes (CEA) du Québec, nous avons choisi de présenter dans cet article la situation des jeunes réfugiés qui fréquentent le CEA de la commission scolaire de Sherbrooke, une région hors de la métropole montréalaise. À partir de leur trajectoire scolaire, nous explorons leur accueil dans le CEA, les facteurs qui influencent leur parcours scolaire, les pratiques institutionnelles mises en œuvre par le monde scolaire à leur égard, les processus suivis à l'intérieur du centre et la manière dont les acteurs institutionnels perçoivent ces jeunes immigrants. Les jeunes rencontrés ont un profil d'apprenants en difficulté ou à risque en raison de leur trajectoire migratoire et scolaire, de leur situation familiale, de leur grand retard scolaire et des traumatismes vécus, mais ils sont résilients au regard des nombreux obstacles situationnels, informationnels, dispositionnels et institutionnels qu'ils rencontrent. Contrairement à la situation des jeunes immigrants montréalais, les jeunes réfugiés en région semblent ainsi utiliser pleinement le contexte et les ressources des CEA comme tuteurs de résilience.

\section{Rattachement des auteures}

${ }^{1}$ Université de Sherbrooke, Sherbrooke, Canada; ${ }^{2}$ Université du Québec à Montréal (UQAM), Montréal, Canada

\section{Correspondance}

marilyn.steinbach@usherbrooke.ca

\section{Mots clés}

jeunes réfugiés; formation générale aux adultes.

\section{Pour citer cet article}

Steinbach, M., Vatz-Laaroussi, M. et Potvin, M. (2016). Accueillir des jeunes réfugiés en région: la formation générale aux adultes comme alternative scolaire? Alterstice, 5(2), 99-108. 


\section{Introduction}

Si plusieurs travaux s'intéressent à l'accueil des réfugiés au Canada et plus spécifiquement au Québec (Arsenault, 2010; Guilbert, 2010; Vatz Laaroussi, 2009), les recherches sont moins nombreuses en ce qui concerne l'insertion et la réussite scolaire des jeunes réfugiés. Pourtant, $8 \%$ des résidents permanents accueillis au Québec chaque année ont le statut de réfugié, et plus de $50 \%$ de ces réfugiés ont moins de 24 ans (MIDI, 2014). De plus, ces jeunes réfugiés connaissent plus souvent des difficultés scolaires que les autres jeunes immigrants, principalement en raison de leur parcours migratoire et scolaire souvent discontinu et semé d'obstacles ou d'événements traumatiques, et en raison de facteurs de défavorisation économique, car les jeunes réfugiés vivent plus souvent dans des familles et des quartiers défavorisés (Kanouté et Lafortune, 2011).

La trajectoire scolaire n'est pas seulement liée au parcours de migration du jeune et de sa famille. Leur installation à Montréal ou en région représente une dimension importante de cette trajectoire. Étant donné les mesures de régionalisation de l'immigration mises en œuvre par le Québec depuis 1993 et du fait que les réfugiés publics ont été les premiers touchés par ces orientations, on assiste à une sur-représentation des familles réfugiées en région comparativement à la région montréalaise, où l'on décompte près de $86,5 \%$ des immigrants du Québec (CMM, 2013). On comprend dès lors que la question des jeunes réfugiés et de leur trajectoire scolaire en région doit être abordée à partir de leurs expériences spécifiques, qui concernent autant les caractéristiques de leur parcours de réfugiés que les spécificités des structures scolaires en région.

La recherche menée par Potvin, Leclercq et collab. ${ }^{1}$ (2014) présente l'originalité de s'intéresser aux jeunes issus de l'immigration de 16 à 24 ans, incluant les réfugiés, qui s'inscrivent en Formation Générale aux Adultes (FGA), que ce soit à la suite d'un passage en classe d'accueil au secondaire ou d'une inscription directe en FGA. Cette recherche visait à retracer leur trajectoire migratoire, sociale et scolaire ainsi que les pratiques institutionnelles mises en œuvre par le monde scolaire à leur égard dans 6 commissions scolaires et 21 centres d'éducation des adultes (CEA) du Québec. L'expérience migratoire est considérée comme un élément important de ces trajectoires et devrait, selon les résultats de l'étude, faire l'objet de plus d'attention et de pratiques différenciées de la part des CEA.

Après une brève présentation de la méthodologie de la recherche en appui, nous nous intéressons dans un premier temps à la trajectoire et aux expériences spécifiques des jeunes réfugiés au Québec puis au contexte régional, pour ensuite aborder la trajectoire institutionnelle qui leur est proposée et finalement identifier les trajectoires scolaires types que ces jeunes suivent. Notre discussion permettra de revenir sur les spécificités de ces jeunes par rapport aux autres réfugiés retrouvés en FGA à Montréal et par rapport à l'ensemble des jeunes immigrants étudiant en FGA. Nous pourrons dès lors poser quelques recommandations pour les commissions scolaires et les centres FGA en région.

\section{Les jeunes réfugiés et la scolarité au Québec}

Trois pour cent ( $3 \%$ ) des résidents permanents au Canada en 2013 sont des jeunes de moins de 15 ans réfugiés (CIC, 2014). On retrouve sensiblement les mêmes proportions au Québec où, sur les $8,6 \%$ de réfugiés (toutes catégories confondues ${ }^{2}$ ) accueillis de 2009 à 2013, $35 \%$ sont des enfants de moins de 15 ans et 22,9\% sont des jeunes de 15 à 24 ans (MIDI, 2014). Malgré cette présence importante, le parcours des jeunes réfugiés au Québec a fait l'objet de peu de recherches spécifiques. Plusieurs études qualitatives permettent cependant de comprendre les difficultés scolaires des jeunes réfugiés en lien avec leur parcours, les traumatismes et les séparations familiales mais aussi avec la vulnérabilité socioéconomique de leur famille, une fois installés au Québec.

\footnotetext{
${ }^{1}$ Cet article repose sur les données d'une recherche financée par le FQRSC et le MELS (Potvin, Leclercq, Steinbach, VatzLaaroussi, Armand, Ouellet et Voyer, 2014).

${ }^{2} 3,3 \%$ sont des demandeurs du statut de réfugié reconnus sur place, $2,8 \%$ des réfugiés publics, 1,6 \% des membres de famille et $0,9 \%$ des parrainés.
} 


\section{Des parcours de mobilité et des difficultés familiales}

Il est bien connu que le parcours migratoire des familles réfugiées au Québec est le plus souvent marqué par des déplacements multiples tant internes avant de quitter le pays qu'externes avant d'arriver au Québec (Vatz Laaroussi, 2009). C'est au cours de ces mobilités répétées que les jeunes vont vivre des séparations avec les parents et la fratrie, et la réunification familiale s'effectue souvent plusieurs mois ou années après l'arrivée d'un parent au Québec (Potvin et Leclercq, 2011). Par ailleurs, plusieurs travaux (Kanouté et Lafortune, 2011; Kanouté et Vatz Laaroussi, 2008) soulignent les difficultés pour de nombreuses familles réfugiées qui disposent d'un capital culturel moins développé à accéder pleinement aux services éducatifs et à comprendre le discours scolaire et les attentes de l'école face à leurs enfants. De plus, ces parents vont être soumis à des conditions d'insertion sociale particulièrement stressantes et à des facteurs situationnels qui ont des impacts sur les parcours scolaires (Potvin et Leclercq, 2014).

\section{Un parcours scolaire chaotique}

Du fait de ces parcours migratoires et familiaux, les jeunes réfugiés ont le plus souvent été amenés à fréquenter plusieurs systèmes scolaires durant leur trajectoire d'exil. C'est le cas des jeunes Afghans dont les familles vont le plus souvent avoir passé plusieurs années en Russie ou au Pakistan avant de s'installer au Québec. Pour les jeunes Congolais, Burundais, Rwandais ou Bhoutanais, c'est au sein des camps de réfugiés qu'ils vont se socialiser à l'école, une école particulièrement chaotique avec de longues périodes d'interruption, des classes de 200 élèves pour un enseignant, des enseignants non formés et des infrastructures insalubres (Vatz Laaroussi, 2015). Dans ces cas, les mobilités successives entraînent, pour les jeunes et leurs familles, la nécessité de développer des adaptations en série dans ce processus d'acculturation, ainsi que des " compétences interculturelles " permettant de passer d'un système à l'autre (Manço, 2002).

L'étude de Papazian-Zohrabian (2013) portant sur les traumatismes de guerre et la maltraitance a démontré la nécessité d'une compréhension dynamique de l'échec scolaire qui distinguerait les difficultés d'apprentissages des difficultés d'ordre psycho-affectif ayant des impacts sur la concentration et l'apprentissage. Les jeunes réfugiés peuvent donc facilement accumuler un grand retard scolaire, lié autant à leur trajectoire scolaire et familiale tumultueuse qu'aux difficultés d'adaptation psychologique, scolaire et sociale à leur nouvelle société. Le retard scolaire a des conséquences à la fois sur le classement du jeune, sur les choix d'orientation scolaire et sur les possibilités de diplomation (Potvin et Leclercq, 2011). Ces retards scolaires et ces difficultés d'ordre psycho-social reliées à la trajectoire spécifique des jeunes réfugiés vont dans plusieurs cas les conduire vers la FGA, soit pour continuer, après 16 ans, des apprentissages scolaires non faits auparavant, soit pour améliorer la maîtrise du français et reprendre les autres matières ensuite. Finalement, notons que ces étudiants réfugiés en FGA seront souvent plus jeunes que les autres apprenants qu'ils côtoieront. Ils auront aussi des histoires et des situations familiales différentes, qui devraient être prises en compte pour faciliter leurs apprentissages et pour mettre en œuvre des services et des modalités pédagogiques qui leur soient adaptées.

\section{Le contexte régional de Sherbrooke : de nombreux réfugiés et des structures en adaptation}

Comparées à la région métropolitaine de Montréal, les régions reçoivent plus d'élèves de première génération, défavorisés, qui arrivent dans le système scolaire québécois après le primaire et qui ont un retard scolaire à l'arrivée au secondaire (Mc Andrew et collab., 2015). À Sherbrooke, le nombre d'immigrants double entre 2000 et 2010 et, en 2012, la population immigrante compte pour près de $7 \%$ de la population de Sherbrooke (Vatz Laaroussi, 2013). Notons également que la ville de Sherbrooke est un pôle d'installation de familles réfugiées parrainées par l'État, si bien qu'environ 50 \% des immigrants de Sherbrooke arrivent avec le statut de réfugié.

Ces spécificités de l'immigration en région, ajoutées à l'augmentation significative de l'effectif estudiantin d'origine immigrante, posent des défis pour les institutions éducatives. Pour les adultes, il y a un seul CEA qui offre les programmes de FGA à Sherbrooke. Dans ce centre, $25 \%$ de l'effectif scolaire est né hors Québec en 2010, soit 834 apprenants sur un total de 3 396. La majorité de ces apprenants, originaires de la Colombie, de l'Afghanistan, 
de l'Irak et de l'Afrique centrale (RDC, Rwanda, Burundi), se trouvent en francisation ${ }^{3}$. Au CEA à Sherbrooke, le directeur est accompagné de trois directeurs adjoints chargés de la répartition des dossiers de direction, cinq conseillers pédagogiques, cinq conseillers en orientation du Service d'accueil, de référence, de conseil et d'accompagnement (SARCA) et de huit secrétaires. De plus, on trouve près de 75 formateurs et formatrices remplissant les fonctions d'enseignement et qui, pour quelques-uns, effectuent les corrections des tests de classement. Un intervenant psychosocial est responsable du volet pré-retour qui vise à sensibiliser et motiver les jeunes pour un retour en formation aux adultes et sur le marché de l'emploi. Comme dans la majorité des écoles et institutions de la région, ce personnel est composé de très peu de personnes d'origine immigrante, bien que ce groupe constitue $25 \%$ de sa clientèle.

\section{Une méthodologie de regards croisés}

Pour l'ensemble de la recherche, nous avons privilégié une approche qualitative, descriptive, comparative et transversale afin d'explorer les facteurs qui influencent les parcours de jeunes arrivant en FGA. Nous avons aussi privilégié une approche biographique (life course), axée sur l'analyse des quatre types de facteurs qui renvoient à des événements prenant en considération différentes sphères de la vie (familiale, scolaire, professionnelle) (Crockett, 2002). Bien que cet article se concentre sur les données recueillies à Sherbrooke, soulignons que l'étude a rencontré dans l'ensemble 184 personnes en entrevue dans 6 commissions scolaires (5 à Montréal, 1 à Sherbrooke) entre janvier 2010 et décembre 2011 (Potvin et collab., 2014). II s'agissait au total de 59 agents institutionnels (responsables de la FGA dans les commissions scolaires, directeurs de CEA et leurs adjoints, responsables des SARCA, conseillers de formation, d'information ou d'orientation, conseillers pédagogiques, travailleurs sociaux, enseignants, directeurs d'écoles secondaire, agents d'Emploi-Québec) et de 80 jeunes de 16 à 24 ans $\left(1^{\mathrm{e}}, 2^{\mathrm{e}}\right.$ et $3^{\mathrm{e}}$ générations) inscrits en FGA et rencontrés dans 24 établissements. Notre objectif était de mettre en œuvre des regards croisés des acteurs de la FGA et des jeunes immigrants qui la fréquentent.

À Sherbrooke, les données ont été recueillies auprès de 24 personnes, soit 12 jeunes issus de l'immigration et 12 intervenants œuvrant en FGA (10 dans un centre d'éducation des adultes, 1 d'Emploi Québec et 1 directrice d'école secondaire). Avec la permission de la commission scolaire, nous avons recruté les intervenants et les apprenants avec la collaboration du directeur du CEA. La quasi-totalité des jeunes (11/12) étaient réfugiés ou parrainés par un membre de leur famille et venant de pays en guerre. Sur ces 12 jeunes ( 6 jeunes hommes et 6 jeunes femmes), la moitié venaient d'Afrique et les autres étaient colombiens (4) et Afghans (2). Quatre étaient arrivés entre 8 et 12 ans, 2 entre 13 et 14 ans et 6 entre 16 et 18 ans. De ce fait, 7 de ces jeunes ont commencé leur scolarité au Québec en classe d'accueil à l'école secondaire, alors que 5 ont commencé directement en FGA. Des entrevues individuelles (d'en moyenne 45 minutes) ont été effectuées avec tous les participants sur place, au CEA, puis ont été entièrement retranscrites. Avec les intervenants, nous avons adopté une démarche d'analyse des pratiques centrée sur les situations, les processus décisionnels et les dispositifs qui s'adressent aux profils de jeunes issus de l'immigration ou réfugiés. Les entrevues avec les jeunes ont cerné les logiques individuelles et structurelles qui orientent les parcours des individus à partir de la reconstitution de moments clés qui structurent leurs parcours. Ce type d'analyse permet de comprendre le cheminement scolaire comme un processus dynamique. L'objectif était de mieux cerner les trajectoires scolaires de ces jeunes réfugiés inscrits en FGA depuis leur arrivée à Sherbrooke.

\section{Les trajectoires institutionnelles}

\section{La FGA et les jeunes réfugiés à Sherbrooke}

Les programmes offerts en FGA par le CEA débutent tous par une session de quatre semaines de soutien pédagogique. Ensuite, le CEA offre les 10 programmes de la FGA, soit : alphabétisation (majoritairement suivis par les immigrants), présecondaire, premier cycle et deuxième cycle du secondaire, intégration sociale, intégration socioprofessionnelle, préparation à la vie professionnelle, préparation aux études postsecondaires et francisation. La population immigrante se trouve majoritaire dans le secteur de la francisation mais elle est aussi distribuée dans

\footnotetext{
${ }^{3}$ La francisation est un programme destiné aux non-francophones adultes. II comprend cinq niveaux : débutant I, débutant II, intermédiaire I, intermédiaire II, avancé.
} 
les cours de $1^{\text {er }}$ et de $2^{\mathrm{e}}$ cycles du secondaire et les sessions préparatoires au monde du travail et aux études postsecondaires. En ce qui concerne ceux qui ont fréquenté le secteur des jeunes au secondaire au Québec, ils ont été envoyés en FGA après 16 ans lorsqu'ils n'avaient pas obtenu les acquis du secondaire 3 :

Ce qu'ils nous disent c'est, comme, ils se rendaient compte qu'au secondaire, au secteur des jeunes, ils ne voyaient pas d'avenir, ils ne voyaient pas le diplôme comme étant possible dans les délais qu'ils avaient. Donc, ils préfèrent venir ici en FGA pour ne pas perdre de temps. (conseillère d'orientation)

\title{
Les articulations institutionnelles externes et internes
}

À Sherbrooke, comme dans les autres centres analysés à Montréal, on constate un manque d'articulation entre les écoles secondaires du secteur des jeunes et les CEA de la FGA, notamment dans le transfert des dossiers des apprenants.

Non, c'est eux autres qui apportent leur bulletin, relevé de note, soit le certificat de naissance soit la carte de résidant permanent soit la carte de citoyenneté... nous autres on ne fait pas affaire avec les écoles pour transférer le dossier. (secrétaire d'accueil)

Même si les conseillers pédagogiques du CEA ont accès aux dossiers scolaires des élèves provenant des classes d'accueil ou de cheminement particulier ${ }^{4}$, la pratique est souvent de faire passer un test de classement aux élèves dès l'inscription du jeune au CEA et sans préparation. Selon plusieurs agents institutionnels rencontrés, cette pratique est systématique lorsque le jeune arrive d'une classe d'accueil, du cheminement particulier ou de l'étranger. De plus, selon les agents institutionnels rencontrés, les enseignants des classes d'accueil ou du cheminement particulier et les directeurs ou intervenants des établissements secondaires contactent rarement le CEA sur les dossiers des élèves qu'ils réfèrent en FGA et ils ne connaissent pas toujours les services (de formation ou complémentaires) que ces jeunes vont y recevoir. II en va de même lorsque les jeunes ont fréquenté d'autres CEA dans différents programmes.

À l'intérieur du CEA, les liens et transitions sont plus fluides. Certains enseignants parlent d'un suivi individualisé des jeunes:

\begin{abstract}
Nous, en francisation, quand l'étudiant a terminé sa francisation, on connaît l'étudiant. On peut faire le suivi. On fait le suivi de nos étudiants qui terminent en francisation ceux dont on connaît un petit peu l'histoire puis on sait qu'ils ont telle problématique, telle difficulté d'apprentissage ou tel problème psycho-social, donc on fait du tutorat auprès de nos étudiants. On les rencontre individuellement. Chaque étudiant a un tuteur. Alors donc on peut faire le suivi alors quand l'étudiant passe de la francisation à la formation générale ou français langue d'enseignement, ben sa fiche de tutorat qui est confidentielle, elle va suivre et nous si on juge important de rencontrer les professeurs on va le faire. (enseignant en francisation)
\end{abstract}

Les liens avec les milieux d'emploi sont très organisés, en particulier avec Emploi-Québec et ses différents programmes de retour aux études, le Carrefour Jeunesse Emploi et la présence active d'une intervenante sociale. Par contre, les élèves réfugiés sont moins nombreux à bénéficier de ces programmes car, en raison d'un plus grand retard scolaire, ils peuvent plus difficilement terminer leur secondaire dans les délais prescrits par ces programmes.

\section{Des services d'accompagnement}

Par ailleurs, ce centre offre au travers du SARCA des services d'accompagnement des étudiants. Trois types de référence peuvent être faites dans un premier temps, et elles le sont souvent en parallèle, selon les profils des jeunes et les programmes de formation qu'ils poursuivent. Ainsi, les candidats rencontrent d'abord un conseiller d'orientation, qui va dresser leur profil de formation et d'orientation professionnelle, et ensuite l'agent du Centre Local d'Emploi, pour demander du financement s'ils sont éligibles. Les étudiants en difficultés d'apprentissage sont plutôt orientés vers le service de soutien à l'apprentissage avant d'être inscrits dans un programme de formation

\footnotetext{
${ }^{4}$ Les cheminements particuliers, au lieu des classes ordinaires, sont offerts aux élèves handicapés ou en difficulté d'adaptation ou d'apprentissage (EHDAA) au secondaire.
} 
spécifique. On constate que les étudiants bénéficient d'un accompagnement avant, pendant et après leur formation, et les intervenants rencontrés estiment que ce mécanisme permet un bon suivi des adultes en formation et leur ouvre des parcours variés correspondant à leurs profils et besoins spécifiques. Cependant, on distingue peu les réalités, expériences et besoins cognitifs, affectifs ou socio-scolaires des immigrants ou des réfugiés de ceux des natifs, sauf pour l'apprentissage de la langue française. On tient peu compte des acquis non formels (comme leur trilinguisme) dans l'évaluation de leurs dossiers et profils de besoin de formation. En particulier, les problématiques psycho-sociales liées au parcours migratoire et aux séparations familiales sont méconnues et les agents vont souvent les référer au Centre de santé et de services sociaux (CSSS) sans suivi spécifique du CEA, ni prise en compte de ces situations dans les apprentissages.

\section{Les discours des acteurs institutionnels}

Les conseillers de formation et d'orientation comme les intervenants en francisation font état de la grande diversité de profils des jeunes immigrants du Centre: certains sont sous-scolarisés, d'autres ont vécu des problèmes psycho-sociaux ou viennent pour apprendre le français, et d'autres encore terminent un ou deux cours du secondaire et diplôment en FGA.

Mais oui, ce sont souvent des jeunes qui ont des problèmes de comportement, qui ont été mis de côté, qui ont été tassés. II y en a d'autres qui ont des problèmes d'apprentissage, qui n'ont pas eu le soutien et le suivi qu'ils devaient avoir. lls ont été mis dans des classes de cheminement particulier temporaire ou de cheminement particulier continu. (enseignant en francisation)

Certains intervenants tendent à voir ces jeunes uniquement sous l'angle de leurs lacunes en français et non de leur parcours migratoire, de leur bagage scolaire, de leurs acquis expérientiels ou de leurs compétences interculturelles et plurilingues. Ils connaissent moins leur trajectoire migratoire et amalgament parfois la faible maîtrise de la langue avec la sous scolarisation.

Maintenant concernant la question des immigrants, moi ce que je constate beaucoup, c'est la difficulté au niveau de la langue. Et ça, ça a des répercussions tout au long de leur cheminement parce que malheureusement ils travaillent en double. S'il ne maîtrise pas bien la langue, comment fait-il pour réussir au même titre qu'un Québécois au régulier? (intervenant social)

Or, pour plusieurs jeunes réfugiés en FGA à Sherbrooke, la FGA représente une opportunité intéressante pour poursuivre leur scolarité et obtenir les connaissances et compétences nécessaires à leur entrée sur le marché de l'emploi.

\section{Le discours des jeunes réfugiés en FGA à Sherbrooke}

\section{Les trajectoires des étudiants en FGA}

Le premier parcours est celui des jeunes arrivés peu avant 16 ans et qui vont d'abord en classe d'accueil au secondaire pour être orientés très rapidement vers la FGA à 17 ans. Le deuxième est celui de jeunes arrivés vers 16 ou 17 ans qui transitent ou non par la classe d'accueil secondaire mais qui sont très rapidement orientés au CEA en francisation. Le troisième est celui des jeunes qui arrivent directement en FGA, par exemple des élèves francophones de 18 ans et plus, n'ayant pas atteint le niveau secondaire 5 avant leur arrivée au Québec. Le quatrième parcours est celui des jeunes arrivés au primaire, qui ont fait la classe d'accueil en primaire et parfois en secondaire, qui n'ont pas réussi à suivre la scolarité régulière et à atteindre à 16 ans le niveau secondaire 2. Ces élèves ont été orientés vers des cheminements particuliers dans le secteur des jeunes et arrivent ensuite en FGA avec des besoins en francisation, en plus d'un rattrapage à faire dans les diverses disciplines. Cette quatrième trajectoire des étudiants rencontrés est la plus inquiétante, on l'appelle la trajectoire chaotique (Potvin et collab., 2014).

\section{Les représentations et parcours scolaires}


Ces étudiants apprécient l'opportunité de travailler individuellement, de manière autonome, et de progresser à leur propre rythme.

En individualisé, c'est mieux. On va selon le rythme de chacun. Ça retient personne. Tout le monde avance à son rythme. (L. P., Colombienne, 19 ans)

Je le vois bien maintenant, c'est une façon pour si tu veux réussir, tu as la clé, mais c'est toi, toi seule. (L. O., Colombienne, 18 ans).

Par contre, plusieurs aimeraient plus de soutien en langue française - soit plus d'enseignement magistral et d'encadrement des enseignants.

Parce qu'il faudra que tu mettes en pratique tout ce que tu as appris quand le prof t'a expliqué en cours magistral pis tu essaies de mettre en application tout ce que tu as appris en français parce que c'est plus difficile. (I. S., Afghan, 17 ans)

La difficulté la plus importante semble être le français écrit/scolaire.

Le français, ça s'améliore pas mal mais c'est quand même compliqué. Parler, c'est facile, mais écrire... ouf ! J'ai pas mal de difficultés mais ça s'améliore. (S. A., Congolais, 18 ans).

Un problème récurrent est la difficulté d'être bien classé, à leur niveau, dans une classe où ils peuvent faire des progrès en partant d'où ils sont. Ces problèmes semblent se poser davantage dans le secteur des jeunes qu'en FGA, où il y a plus de souplesse dans les classements.

Moi je l'ai constaté moi-même parce que c'était évident, mais quand j'ai parti de là-bas j'ai vraiment remarqué mon retard en français quand je suis venu ici, j'étais classé en $2^{\mathrm{e}}$ français quand je suis arrivé ici après 2-3 mois, le prof, mon prof de français, a constaté que je n'étais pas très bon, ... il m'a demandé de recommencer mon parcours en français » (I. S., Afghan, 17 ans)

Dans ces 12 entrevues, on voit que les étudiants apprécient d'être finalement classés au niveau où ils sont actuellement, pour être capables de réussir, de comprendre et de faire des progrès.

Je commence à zéro en français, en maths à zéro, en anglais j'étais en 1. J'ai commencé puis ... en tout cas pour moi, c'était une vraiment une formation. J'ai vraiment aimé ça. (E. W., Tanzanien, 16 ans).

La moitié (6/12) de ces apprenants est passée par le cheminement particulier (CP) et, avec le recul, ces derniers sont convaincus qu'ils ont été mal évalués : ils n'avaient pas de " trouble d’apprentissage " mais ne maîtrisaient pas la langue ou étaient sous-scolarisés. On note, pour ces jeunes et pour leur famille, un manque important d'information sur les raisons de ce passage en CP et sur ce qu'ils pouvaient en attendre. Les jeunes passés par les classes de CP ont connu, pour la plupart, une trajectoire migratoire particulièrement difficile de réfugiés avec expérience de guerre, de violences, de séparations et de pertes familiales, de vie en camps de réfugiés, ou avec plusieurs déplacements. Ces jeunes expriment des sentiments de frustration et de découragement lorsqu'ils expliquent leur trajectoire en $\mathrm{CP}$, et le malaise qu'ils avaient à être placés avec des jeunes vraiment démotivés et parfois violents, souvent avec des troubles de comportement.

J'ai peut-être pas réussi l'examen mais pourquoi on me met dans une classe des troubles du comportement? Les classes de cheminement, là-bas c'est du monde qui ont un trouble de comportement. Moi j'en avais aucun, puis on me met là-dedans, c'est ça que je ne comprenais pas. (E. W., Tanzanien, 16 ans)

Selon ce jeune, on ne lui aurait jamais vraiment expliqué pourquoi on l'orientait en CP :

J'ai jamais osé demander puis ce que je trouve aussi le plus dur aussi pour les parents, eux autres n'ont pas le droit de dire ok c'est bon. Elle-même elle ne sait pas c'est quoi. C'est comme il faut vraiment que toi-même tu ailles vraiment essayer de te renseigner sur les affaires comme ça. (E.W., Tanzanien, 16 ans) 
On perçoit, chez eux, un sentiment d'injustice d'avoir été mis dans une « voie de garage » et on constate le manque de services adaptés pour les élèves sous-scolarisés dans le système des jeunes. Par exemple, un élève explique sa démotivation envers l'école parce qu'il a été placé en cheminement particulier :

Aucune envie d'aller à l'école parce que tu trouves que tu n'apprends rien. Tu sais qu'en cheminement, les profs ne vont pas t'apprendre grand-chose à part faire des stages, couper du bois, faire un peu de français... II y a pas vraiment assez de journées à l'école et tu n'apprends absolument rien. (S. A., Congolais, 18 ans)

Ils parlent peu de leurs conditions de vie en dehors de l'école. Or tous viennent de familles socio-économiquement défavorisées, sans exception. La moitié était sous-scolarisée à cause des circonstances politiques. Évidemment, ces circonstances de vie avant et après la migration affectent énormément leurs trajectoires scolaires, et on trouve en FGA en région une population peu représentative de l'ensemble des étudiants immigrants. De plus, contrairement aux élèves immigrants du secteur des jeunes, ces jeunes ne parlent jamais des problèmes (sociaux, de communication) entre étudiants d'origine immigrante et étudiants d'origine québécoise. Ils ont l'impression que la majorité des étudiants au centre sont d'origine immigrante, ce qui n'est pas le cas à Sherbrooke.

\section{Discussion}

Les différents acteurs rencontrés ont parfois des représentations différentes de la FGA. Pour les uns, ce type de formation, par sa souplesse, son adaptation au profil scolaire des adultes et son approche individualisée et d'autoformation assistée, permet de développer l'autonomie et l'estime de soi des apprenants, leur offrant ainsi de bonnes conditions d'apprentissage. Pour d'autres, cette approche, en raison du trop grand nombre d'apprenants par classe et des classe multi-niveaux, ne favorise pas les échanges fréquents enseignant-apprenant, ni entre les apprenants. Les étudiants rencontrés ont des représentations positives de la FGA, et souvent un sentiment de sortir de l'échec vécu à l'école. Ils apprécient les classes pluriculturelles, et ne font pas mention de relations conflictuelles, ni de problèmes de ségrégation ou discrimination souvent vécus dans le secteur des jeunes (Steinbach et Grenier, 2013). Plusieurs étudiants constatent par contre une non-reconnaissance de leurs capacités, et la structure des situations d'apprentissage en FGA ne semble pas adaptée aux étudiants réfugiés. Les problèmes abordés portent aussi sur le fait d'avoir été placés en cheminement particulier dans le secteur des jeunes. On retrouve ce même enjeu dans le discours des autres apprenants des CEA sur l'île de Montréal (Potvin et collab., 2014).

L'analyse des parcours des jeunes réfugiés de Sherbrooke permet d'identifier un groupe important de jeunes au cheminement perturbé et problématique, parsemé de difficultés scolaires et de problèmes familiaux, ayant souvent un parcours migratoire lourd et traumatisant. À Sherbrooke, la trajectoire dite chaotique s'applique aussi aux jeunes arrivés beaucoup plus tôt, entre 8 et 12 ans, provenant de pays en guerre et de camps de réfugiés, peu ou pas scolarisés avant leur arrivée. Ces jeunes passent par les classes d'accueil au primaire puis au secondaire ou en classes de cheminement particulier. Leur cheminement scolaire est chaotique, ils ont peu de contrôle sur ce qui leur arrive et ils se retrouvent en FGA, où ils apprécient la personnalisation du suivi et la possibilité de reprendre leurs apprentissages à la base sans être stigmatisés. Ils continuent à avoir de grands rêves d'avenir, ont besoin de soutien psycho-social et de temps pour continuer leurs apprentissages. Ils sont surtout africains et afghans. Ces apprenants au parcours chaotique sont une exception à la norme, car les jeunes issus de l'immigration qui arrivent plus jeunes ont souvent une meilleure intégration et réussite scolaire (Mc Andrew et collab., 2015), tandis que leurs compatriotes qui arrivent plus tard semblent avoir plus de difficultés dans le système éducatif au Québec. Notre analyse des spécificités des étudiants de parcours chaotique souligne l'importance de prendre en considération des éléments du parcours migratoire des familles mais aussi le type de contexte régional et scolaire dans lequel ils s'inscrivent. 


\section{Conclusion}

La recherche a mis en évidence l'importance de prendre en compte les facteurs de risque, les réalités et les parcours de ces jeunes afin d'assurer un meilleur ciblage des interventions (Potvin et collab., 2014). Les agents institutionnels témoignent d'un travail d'adaptation en cours de la FGA en région mais qui se vit dans des conditions précaires, un manque de ressources professionnelles et des logiques systémiques mouvantes. Leurs discours insistent sur les difficultés et les besoins linguistiques et psychopédagogiques spécifiques des jeunes réfugiés.

Les données vont à l'encontre de l'image de décrocheurs souvent attribuée par les médias aux jeunes en FGA. Les jeunes rencontrés ont un profil d'apprenants en difficulté ou à risque mais sont résilients au regard des facteurs de risque, dont la situation de grand retard scolaire en raison de facteurs situationnels (expérience migratoire, scolarité chaotique, écarts entre systèmes, faible francisation), informationnels (méconnaissance du système), dispositionnels (arrivée à l'adolescence difficile, démotivation à apprendre le français) et institutionnels (tests de classement, services ou soutiens inadaptés). Les jeunes réfugiés en région semblent d'ailleurs utiliser pleinement le contexte et les ressources de la FGA comme tuteurs de résilience (Vatz Laaroussi, 2009 et 2015). Par contre, les milieux institutionnels tout comme les jeunes soulignent l'importance de mettre en œuvre des mesures et des structures souples et personnalisées permettant de favoriser la résilience et la réussite.

Ainsi, il serait important que les jeunes réfugiés arrivés au secondaire puissent bénéficier d'une continuité de services, d'une articulation des programmes, d'accompagnement et de passerelles facilitant la transition entre les deux secteurs (jeunes et adultes). Par ailleurs, il est indispensable de prendre en compte les réalités migratoires, familiales, scolaires, identitaires et sociales des jeunes adultes réfugiés dans l'évaluation de leurs dossiers et de leurs acquis. De même, certaines pratiques, programmes de formation ou soutiens financiers devraient être plus flexibles et accessibles, et liés aux réalités de ces apprenants plutôt qu'à des règles comptables. Enfin, il est clair que les approches pédagogiques interactives, participatives et coopératives, de tutorat entre pairs, sont les plus pertinentes et appréciées des jeunes. Il est important de les développer et de former les intervenants en FGA à ces méthodes et approches. Cette formation fait particulièrement défaut en région, et il y a urgence à la structurer, en amenant les intervenants des régions à fréquenter des formations données à Montréal et en décentrant les lieux de formation vers les villes moyennes comme Sherbrooke et les commissions scolaires régionales où les jeunes réfugiés continuent à arriver de manière accélérée.

\section{Références}

Arsenault (2010). Les réfugiés colombiens au Québec : des pratiques transnationales centrées sur la famille. Lien social et politiques, 64, 51-64.

Citoyenneté et immigration Canada (2014). Faits et chiffres 2013 - Aperçu de l'immigration : Résidents permanents. http://www.cic.gc.ca/francais/ressources/statistiques/faits2013/index.asp

Communauté métropolitaine de Montréal (CMM) (2013). Bulletin de la communauté métropolitaine de Montréal [En ligne]. Perspective, 24. http://cmm.qc.ca/fileadmin/user_upload/periodique/24_Perspective.pdf

Crockett, L. J. (2002). Agency in the life course: Concepts and processes, agency, motivation, and the life course. Lincoln : University of Nebraska Press.

Guilbert, L. (2010). Paroles de femmes immigrantes et réfugiées à Québec : les dynamiques familiales dans une ville universitaire moyenne, dans : N. Lacasse et J. Palard (dir.), Immigration et intégration au Québec et en France : Enjeux de société et expériences territoriales (p. 103-123). Québec : Presses de l'Université Laval.

Kanouté, F. et Lafortune, G. (dir.) (2011). Familles québécoises d'origine immigrante. Les dynamiques de l'établissement. Montréal : Presses de l'Université de Montréal.

Kanouté, F. et Vatz Laaroussi, M. (2008). La relation écoles-familles immigrantes : une préoccupation récurrente et pertinente. Revue des sciences de l'éducation, 34(2), 259-264. 
McAndrew, M. Balde, A., Bakhshaei, M., Tardif-Grenier, K., Armand, F., Ledent, J., Potvin, M., Guyon, S., Lemieux, G., Vatz-Laaroussi, M., Audet, G., Carpentier, A. et Rousseau, C. (2015). La réussite éducative des élèves issus de l'immigration. Dix ans de recherche et d'interventions au Québec. Montréal : Presses de l'Université de Montréal.

Manço, A. (2002). Compétences interculturelles des jeunes issus de l'immigration. Perspectives théoriques et pratiques. Paris : L'Harmattan.

Ministère de l'Immigration, de la Diversité et de I'Inclusion (MIDI) (2014). Portraits statistiques, L'immigration permanente au Québec selon les catégories d'immigration et quelques composantes 2009-2013. http://www.midi.gouv.qc.ca/publications/fr/recherches-statistiques/Portraits_categories_2009-2013.pdf

Papazian-Zohrabian, G. (2013). Le deuil traumatique chez l'enfant et son influence sur la construction de son identité. Revue québécoise de psychologie, 34(2), 83-100.

Potvin, M. et Leclercq, J.-B. (2011). Histoires migratoires et scolaires de jeunes à l'éducation des adultes. Dans Fasal Kanouté et Gina Lafortune (dir.) Familles québécoises d'origine immigrante. Les dynamiques de l'établissement. (p. 129-144). Montréal : Presses de l’Université de Montréal.

Potvin, M. et Leclercq, J.-B. (2014). Facteurs affectant la trajectoire scolaire des jeunes de 16-24 ans issus de l'immigration en formation générale des adultes. Revue des sciences de l'éducation, 40(2), 309-350.

Potvin, M., Leclercq, J.-B., Steinbach, M., Vatz-Laaroussi, M., Armand, F., Ouellet, C. et Voyer, B. (2014). Les jeunes de 16-24 ans issus de l'immigration à l'éducation des adultes : parcours, processus de classements et orientation scolaires (Rapport scientifique intégral 2009-PE-130938 pour le Programme des Actions concertées Persévérance et réussite scolaires, Fonds québécois de recherche sur la culture et la société (FQRSC) et son partenaire, le ministère de l’Éducation, du Loisir et du Sport (MELS).

Steinbach, M. et Grenier, N. (2013). « Nous autres aussi on aimerait ça garder notre culture » : les attitudes des élèves de la société d'accueil envers les élèves issus de l'immigration. McGill Journal of Education, 48(1), 183-202.

Vatz Laaroussi M. (2009). Mobilités, réseaux et résilience : le cas des familles immigrantes et réfugiées au Québec. Québec : Presses de I’Université du Québec.

Vatz Laaroussi, M. (2013). Écriture et histoires familiales de migration (rapport final pour le MELS et le FQRSC). http://www.elodil.umontreal.ca/fileadmin/documents/Recherche/Histoires-familiales-EHF/Rapports/vatzecriture-histoires-familiales-rapport-2013.pdf

Vatz Laaroussi, M. (2015). Les rapports intergénérationnels dans la migration. De la transmission au changement social. Québec : Presses de l’Université du Québec. 